\title{
A DETERMINATION OF SECULAR PARALLAXES OF REFERENCE STARS FROM RELATIVE PROPER MOTIONS OF OPEN CLUSTERS
}

\section{A. N. Deutsch \\ Pulkovo Observatory}

The determination of secular parallaxes of stars is usually based on meridian observations of proper motions of bright stars, this introducing known systematic errors. The mean parallaxes of stars can be obtained by means of radial velocities which are known for the bright stars. The more perspective method, the reference of stars to galaxies, is not applicable at low galactic latitudes.

It is therefore interesting to attempt a determination of the secular parallaxes by means of known photometric distances of open clusters. For this purpose it is necessary to know the relative proper motion of the clusters.

The method is easily understood from the figure.

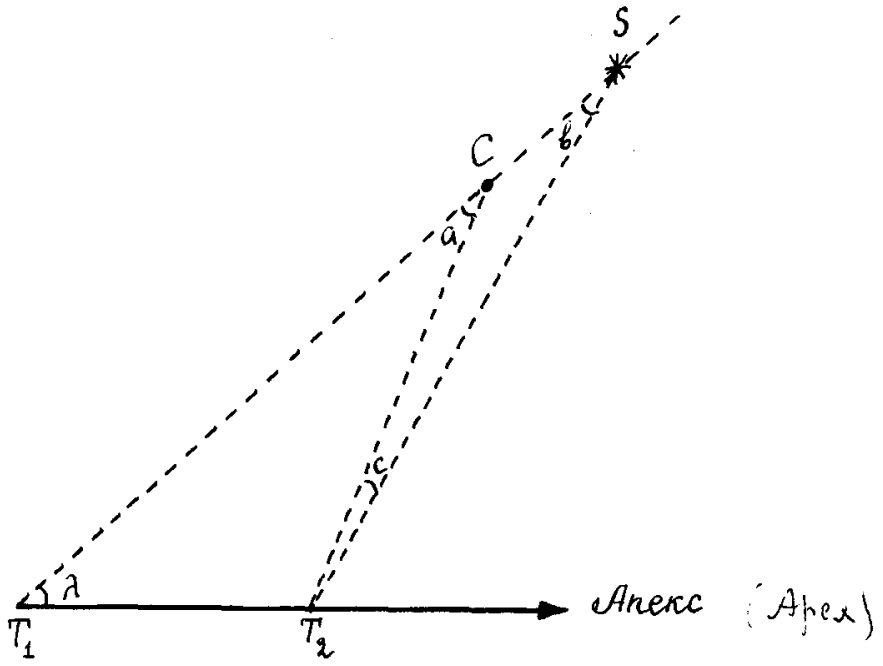

The points $\mathrm{T}_{1}$ and $\mathrm{T}_{2}$ represent two positions of the Sun which has a peculiar relocity of $19.5 \mathrm{~km} / \mathrm{sec}$. The directions from these points to the cluster $\mathrm{C}$ and the group of reference stars $S$ form parallactic angles $a$ and $b$. The ingle $\mathrm{c}$ is the difference between the parallactic angles $\mathrm{a}-\mathrm{b}$. The angle is ound from

$$
a^{\prime \prime}=\frac{v_{\odot} \sin \lambda}{4.74 \cdot \mathcal{L}}
$$


where $\mathrm{V}_{\odot}+19.5 \mathrm{~km} / \mathrm{sec}, \mathrm{r}$ - the distance to the cluster in parsecs. The angle $c$, which is found from observations and related to a one-year inter$\mathrm{val}$, is the $v$ component of the proper motion of the cluster relative to the reference stars. This angle is found from the following equation:

$$
U=\mu_{x} \sin x_{y}+\mu_{y} \cos x
$$

where $\chi$ is the position angle of the antiapex. The angles $X$ and $\lambda$ are determined from $m_{\text {the }}$ corresponding polar triangle, adopting the standard solar apex $\mathrm{A}=18^{\mathrm{h}}, \mathrm{D}=+30^{\circ}$.

For each cluster we obtain the angle $b=a-c$, which includes the peculiar component of motion of the cluster in the direction of the apex or antiapex. It is assumed that the sum of these components for all the considered clusters is equal to zero.

The secular parallax q of the reference stars is found from the formula

$$
q \sin \lambda=b
$$

and by the method of least squares

$$
\bar{q}=\frac{\sum p b \sin \lambda}{\sum p b \sin ^{2} \lambda}
$$

where $p$ is the weight of each equation.

Only clusters closer than about 2000 pcs with sufficiently accurate relative proper motions were used for the investigation. There are thirtyfour such clusters, seven of which have proper motions determined by two or more authors. The observational material differs in number of measured plate pairs, the epoch difference and the focal-length of the telescope used. Therefore the determination of weights was not a simple task. The best proper motions were assigned weight five. These include the proper motions of five clusters: NGC 1907, 1912, 2168, 6882 and 6885 determined from 4 5 plate pairs and an epoch difference of $55-57$ years. The proper motions of these clusters were determined by V.V. Lavdovsky at Pulkovo, using the Cartf-du-Ciel astrograph and have a probable error of $\pm 0 !: 0004$ on the average . The least accurate determinations were assigned weight one.

The mean secular parallax of the reference stars of photographic magnitude 12.7 were found from the formula (3)

$$
\bar{q}=0 ! \cdot 0041 \pm 0 ! \cdot 0009
$$

for the mean galactic latitude $5^{\circ}$. 
This result was obtained using distances determined for the thirtyone clusters by various authors.

If one adopt the distances for the clusters as given by H. Johnson ${ }^{2}$, who found that $R=\frac{A}{E_{R-V}}$ varies with galactic longitude, the secular parallax equals $0: 0053 \pm 0 ! 10609$ ( prob. err. ) for the mean galactic latitude $3^{\circ}$ and $m=12.7$. In this latter determination the cluster NGC 2682 with $b=32^{\circ}$ was excluded and three additional clusters added as these according to Johnson are located closer than 2000 psc.

The secular parallaxes as derived by different authors are given in the following table

Table 1

\begin{tabular}{lcc}
\multicolumn{1}{c}{ Authors } & $\begin{array}{c}\text { Secular } \\
\text { parallaxes }\end{array}$ & $\begin{array}{c}\text { Years of } \\
\text { publication }\end{array}$ \\
van Rhijn and Bok & $0 ! 0062$ & 1931 \\
Binnendijk & 0.0066 & 1943 \\
Deutsch & 0.0104 & 1947 \\
Vyssotsky and Williams & 0.0065 & 1948 \\
Luyten & 0.0110 & 1965
\end{tabular}

If one takes into account that stars with large proper motions $\mu \geq 0$ !'05 are excluded from reference stars, then a correction to $\bar{q}$ equal to 0 !:0010 can be added. The final value of $\vec{q}$ is then $0 ! \cdot 0063$, which is in good agreement with the data of the table.

References:

1) V. V. Lavdovsky, Izwestia Pulkvo Observ, no. 176, 1965.

2) H. L. Johnson, "Stars and Stellar Systems", vol. 7, p. 216, 1968. 